\title{
Comprensión lectora y hábitos de estudio en estudiantes de educación superior, desde el aprendizaje conceptual y procedimental
}

\section{Reading comprehension and study habits in higher education students, from conceptual and procedural learning}

DURÁN-FLORES, Ma. Nelly†๋*, FARIAS-BRACAMONTES, Juan Carlos, GARCÍA-GARCÍA, Jesús Alberto y CU-FARFÁN-LÓPEZ, Julio

Universidad Autónoma de Coahuila-Facultad de Ciencia, Educación y Humanidades

ID $1^{\mathrm{er}}$ Autor: Ma. Nelly, Durán Flores

ID $1^{\text {er }}$ Coautor: Juan Carlos, Farías-Bracamontes / ORC ID: 0000-0003-0939-3608, CVU CONACYT ID: 371500

ID $2^{\text {do }}$ Coautor: Jesús Alberto, García-García / ORC ID: 0000-0003-1369-311X, Researcher ID Thomson: D-8211-2019, CVU CONACYT ID: 568438

ID $3^{\text {er }}$ Coautor: Julio, Cufarfán-López / ORC ID: 0000-0001-7203-8022

DOI: $10.35429 /$ JET.2020.12.4.21.27

Recibido: 05 de Septiembre, 2020; Aceptado 20 de Diciembre, 2020

\section{Resumen}

Este reporte de investigación aborda un estudio respecto al el efecto de la comprensión lectora y los hábitos de estudio utilizados por alumnos de educación superior en relación con el proceso de aprendizaje de contenido conceptual y procedimental El objetivo de la investigación fue conocer la importancia y el impacto de los hábitos de estudio en el aprendizaje procedimental y conceptual. El enfoque del estudio fue cuantitativo, con alcances exploratorios. El diseño de investigación consta de tres variables complejas y 71 variables simples, las variables simples corresponden a un nivel de medición numérico de razón. El cuestionario cuenta con cinco páginas compuestas de 71 preguntas divididas en tres bloques, utilizando una escala tipo Likert. La muestra estuvo conformada por 200 estudiantes de educación superior, el $28.5 \%$ está conformado por hombres y el $51 \%$ por mujeres. El área de aprendizaje está relacionada fuertemente con el de comprensión lectora y hábitos de estudio, mientras estudiante siente satisfacción al estudia, y que, le gusta trabajar en equipo, asistir a la biblioteca, y estar satisfecho al incrementar su promedio académico. El hábito de estudio está fuertemente vinculado con el aprendizaje conceptual y procedimental.

Comprensión lectora y hábitos de estudio en estudiantes de educación superior, Desde el aprendizaje conceptual y procedimental

\begin{abstract}
The This research report addresses a study regarding the effect of reading comprehension and study habits used by higher education students in relation to the learning process of conceptual and procedural content. The objective of the research was to know the importance and impact of study habits in procedural and conceptual learning. The focus of the study was quantitative, with exploratory scopes. The research design consists of three complex variables and 71 simple variables, the simple variables correspond to a numerical level of ratio measurement. The questionnaire has five pages made up of 71 questions divided into three blocks, using a Likerttype scale. The sample consisted of 200 higher education students, $28.5 \%$ were men and $51 \%$ women. The area of learning is strongly related to reading comprehension and study habits, while the student feels satisfaction in studying, and that he likes to work in a team, attend the library, and be satisfied by increasing his academic average. The study habit is strongly linked to conceptual and procedural learning, among others.
\end{abstract}

Reading comprehension and study habits in higher education students, From conceptual and procedural learning

\footnotetext{
* Correspondencia del Autor (Correo electrónico: carlos.farias@uadec.edu.mx)

$\dagger$ Investigador contribuyendo como primer autor.
} 


\section{Introducción}

El presente trabajo aborda un estudio de la comprensión lectora y hábitos de estudio en estudiantes de educación superior, desde el aprendizaje conceptual y procedimental. El proceso de aprendizaje centrado en el alumno toma en cuenta, entre otros, el contenido conceptual y el procedimental como acciones donde el sujeto tiene control y responsabilidad para construir su propio conocimiento. En dichas relaciones, el aprendizaje de contenido tiene su expresión en la comprensión y de ella en la comprensión lectora que acerca al sujeto con el conocimiento que este busca. Por su parte, el aprendizaje procedimental, a partir de los hábitos de estudio permite al hombre facilitar su acceso al conocimiento, resultando como una estrategia y acción concreta.

Es importante mencionar que aun y cuando existen diferentes estrategias de lectura, los estudiantes de nivel medio superior no cuentan con las herramientas necesarias para poner en práctica dichas estrategias. El reporte de la investigación tiene como objetivos conocer la importancia y el impacto de los hábitos de estudio en el aprendizaje procedimental y conceptual, desarrollar estrategias de aprendizaje conceptual y procedimental como herramientas en el proceso educativo, conocer y aplicar los diferentes métodos de comprensión lectora en el aprendizaje conceptual y procedimental, proponer cambios en la estrategia de aprendizaje que mejoren la comprensión lectora y los hábitos de estudio de estudiantes de primer año de educación superior, para obtener mejores resultados académicos.

También pretende validar un modelo estructural de la comprensión lectora y hábitos de estudio mediante un diseño ortogonal, para aportar herramientas para el desarrollo del aprendizaje conceptual y procedimental en estudiantes de educación superior. Se muestran investigaciones realizadas sobre Aprendizaje desde distintos aportes didácticos y/o metodológicos de la enseñanza, no solamente como un proceso educativo, aborda también las motivaciones que influyen en la vida académica de los estudiantes universitarios, así mismo se muestran investigaciones que hablan sobre los hábitos de estudio, mencionando que existen correlaciones estadísticamente significativas del rendimiento académico sólo con algunos componentes del hábito lector, como la actitud frente a la lectura.
Finalmente se incluyen revisiones sobre comprensión lectora en donde se describen las estrategias metacognitivas aplicadas en la escritura y comprensión lectora, diseños de un programa para mejorar la comprensión de textos en estudiantes universitarios, entre otros También se describe el instrumento que empleo para la recolección de los datos, las pruebas de confiabilidad, las variables y la muestra, así como el desarrollo y ejecución de la investigación, sus etapas de lectura y presentación.

En primera instancia se analizan las propiedades psicométricas del instrumento mediante las pruebas de confiabilidad y validez. Por otro lado, se analiza el comportamiento de las variables mediante análisis descriptivos. Las pruebas de hipótesis con análisis comparativos de la muestra. Se establecieron los correlatos entre las dimensiones y los modelos de regresión lineal. Por último, se exploró la estructura factorial de las variables y del instrumento que integran. La principal aportación de este estudio es en sí una primera propuesta validada en su contenido para evaluar la comprensión lectora, los hábitos de estudio y el aprendizaje conceptual y procedimental de una forma más completa, por lo que se encontrará con alto atractivo las premisas y planteamientos vertidos en este producto.

\section{Metodología de estudio}

Para esta investigación se utilizaron 71 variables de tipo nominal, separadas en 3 apartados utilizándose el tipo de escala de Likert; con un nivel de medición de: totalmente en desacuerdo, bastante en desacuerdo, ni acuerdo ni desacuerdo, bastante de acuerdo y totalmente de acuerdo; nunca, casi nunca, algunas veces, casi siempre y siempre; nada, poco, bastante, mucho y siempre. La muestra resulto de 200 estudiantes de Educación Superior cuestionados con las siguientes características: 5 estudiantes del sexo femenino y 5 del sexo masculino, que cursen el primer semestre de educación superior de entre 16 y 20 años, Tipo de muestreo estadístico, era probabilístico porque todos tienen, la misma oportunidad de participar. El contexto escolar en el que se encuentra sumergida la institución, pertenece a un nivel socioeconómico regular, y que a la vez está rodeado de un ambiente de estudiantes locales y foráneos. Los criterios de exclusión fueron: que no fueran estudiantes de educación superior, más de 20 años. 
La validez de contenido del instrumento se obtuvo en primera instancia mediante dos rondas de jueceo con expertos en el contenido y temáticas abordadas en la investigación. Las recomendaciones que se me hicieron fueron; revisar bibliografía que ellos consideraron importante. Las modificaciones fueron: disminución de reactivos, por considerarse que algunos no eran pertinentes y modificar la redacción en algunos puntos.

La fiabilidad se obtuvo mediante el método de consistencia interna expresado con el coeficiente alfa de Cronbach. De acuerdo con los resultados anteriores, se conoce que el instrumento tiene validez en el primer apartado titulado "aprendizaje", mientras que los dos restantes requieren de una reestructuración por no haber pasado la prueba de validación, según el Alfa de Cronbach.

\section{Resultados}

Para dar respuesta a la interrogante a partir de la organización de la información recabada a través del instrumento de investigación, se procedió a tratar estadísticamente los datos: mediante la caracterización - estadística descriptiva, análisis comparativo, análisis correlacional y análisis factorial, para la detección de estructuras subyacentes referentes al ámbito de la investigación y su integración de resultados.

Se exploró la fiabilidad del instrumento como propiedad psicométrica mediante el método de consistencia interna Alfa de Cronbach, el total de la escala obtuvo un coeficiente de 913. La consistencia interna de los tres apartados del instrumento se describe en la siguiente tabla.

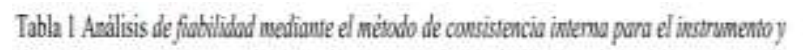
sus apartadas

\begin{tabular}{ccc}
\hline Conjunto & Elementos & Alfa de Cronbach \\
\hline Comprensión lectura & 16 & .674 \\
Aprendizaje & 23 & .794 \\
Habitos de estudio & 24 & .899 \\
Instrumento & 71 & 913 \\
complete & & \\
\hline
\end{tabular}

El total de la muestra estuvo conformado por 200 estudiantes de primer semestre de Educación Superior de la Universidad Autónoma de Nuevo León, el $28.5 \%$ fueron hombres y un $51 \%$ por mujeres, lo que indica una muestra homogénea.

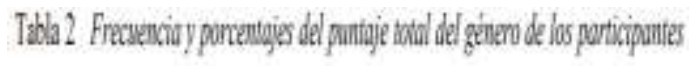

\begin{tabular}{|c|c|c|c|c|}
\hline Generv & Freuenciat & Partentivi & $\begin{array}{c}\text { Poncantaik } \\
\text { sulido }\end{array}$ & $\begin{array}{l}\text { Pancentioje } \\
\text { acumulad }\end{array}$ \\
\hline Hombre & 57 & 28.5 & 28.5 & 28.5 \\
\hline Myjer & [43 & & 71.5 & 1000 \\
\hline Total & 200 & 100.0 & 1000 & \\
\hline
\end{tabular}

Al realizar el análisis de la edad de los encuestados, se encontró que oscilaba entre los 16 y 26 años, resultando ocho grupos, en el .5\% de la muestra se encuentran estudiantes de 16, 22 y 26 años, el mayor número es el $40 \%$ de 17 años, seguido por un $39 \%$ de 18 años, un 14.5 de 19 años el $4.0 \%$ y $1.0 \%$ corresponden a 21 y 22 años respectivamente. Lo que indica que el mayor porcentaje de estudiantes de primer semestre de Educación Superior se concentra entre los 17 y 18 años.

\begin{tabular}{|c|c|c|c|c|}
\hline Edad & Frecuencia & Porcentaje & Porcentraje nifido & Porcentuje scumulado \\
\hline 16 & 1 & 5 & 5 & 5 \\
\hline 17 & 80 & 400 & 40.0 & 40.5 \\
\hline 18 & 78 & 39.0 & 39.0 & 79.5 \\
\hline 19 & 29 & 145 & 14.5 & 94.0 \\
\hline 20 & 8 & 40 & 4.0 & 980 \\
\hline 21 & 2 & 10 & 1.0 & 99.0 \\
\hline 22 & 1 & 5 & 5 & 99.5 \\
\hline 26 & 1 & 5 & 5 & 100.0 \\
\hline Total & 200 & 1000 & 100.0 & \\
\hline
\end{tabular}

\section{Conclusiones}

El propósito de esta tesis era conocer, cuál era el efecto de la comprensión lectora y los hábitos de estudio utilizados por alumnos de educación superior, en relación con el proceso de aprendizaje de contenido conceptual y procedimental. Para dar respuesta a la interrogante, se procedió a tratar estadísticamente los datos recabados a través del instrumento de investigación, el cual resultó con un coeficiente de fiabilidad de .913, mediante el método de consistencia interna Alfa de Cronbach. La muestra indicó ser homogénea, conformada por 200 participantes, el $28.5 \%$ fueron hombres y el $71.5 \%$ por mujeres, con un rango de edad de entre 16 y 26 años, encontrándose el mayor porcentaje entre 17 y 18 años, el $99.5 \%$ solteros y sin hijos. En la prueba de adecuación muestral se encontró una medida de .848 considerada como un nivel de adecuación ideal. 
Sin embargo, la prueba de esfericidad de Bartlett, presento una distribución entre las categorías $(\mathrm{X} 2=5467.137, \mathrm{gl}=1953$, sig. $=.000)$ La hipótesis se rechaza por ser menor a .050 , el resultado fue de .000 , lo cual muestra que la matriz de correlaciones es adecuada para la factorización. El método de extracción fue el de componentes principales y el de rotación el de normalización Varimax con Kaiser, para darle mayor claridad a la solución. A partir de los resultados obtenidos se concluye que para el objetivo uno de la investigación, que propone conocer y aplicar los diferentes métodos de comprensión lectora en el aprendizaje conceptual y procedimental, se encontró que, los indicadores con medias altas son los que se refieren a lo piensan los estudiantes de ser buenos o buenas en las tareas escolares e imaginar fácilmente las situaciones que leen, así como el gusto por leer. Las variables con una media menor tienen que ver con las dificultades que tuvieron cuando aprendieron a leer.

El nivel de significancia utilizado para la prueba de hipótesis fue de $\mathrm{p} \leq .050$. No hay diferencias significativas por sexo en la variable comprensión lectora, la media fue igual en ambos grupos, el valor de esta variable se encuentra a cero puntos del valor máximo posible para esta escala.

Esto demuestra que las mujeres, tienen igual nivel de comprensión lectora que los hombres, resultando un $95 \%$ de intervalo de confianza. Del análisis comparativo se encontró que existen diferencias significativas por sexo en la mayoría de las variables, posiblemente porque las participantes son más mujeres que hombres.

En el eje de comprensión lectora en tres de las variables la diferencia es significativa hacia las mujeres en relación con entender lo que leen. Se encontraron correlaciones significativas de signo positivo, resultando que, el estudiante de educación superior entre más comprende lo que lee, obtiene un aprendizaje más significativo. Asimismo, la satisfacción personal también se relaciona de manera proporcional con obtener mejores resultados en su calificación.

Cabe destacar que el nivel de las correlaciones entre las variables mencionadas se considera débil. El resultado del análisis factorial exploratorio en el segundo componente se integró con doce reactivos, que explican el 8 $\%$ de la varianza. Este componente se etiquetó con el nombre de técnicas de estudio.
Dichas técnicas consisten en elaboración de mapas conceptuales, esquemas de contenidos de temas, resaltar el texto. La habilidad lectora, etiqueta que se asignó al tercer componente de la estructura, mide la facilidad con la que aprende, comprende lo que lee, imaginar situaciones al leer, máximo provecho intelectual.

Este componente explica el $7 \%$ de la varianza y se integró por nueve reactivos Para dar respuesta al objetivo dos referente a conocer la importancia y el impacto de los hábitos de estudio en el aprendizaje procedimental y conceptual, se encontró que en el análisis descriptivo, los indicadores con medias altas son los que se refieren a que ellos consideran que aprobar o no las asignaturas del curso depende de su esfuerzo personal y así como, les gusta que los contenidos del tema siempre se trabajen con mapas conceptuales y las variables con una media menor tienen que ver con lo que piensan en que sacar mejor o peor nota en los exámenes depende más de la suerte que de su propio esfuerzo. Por otro lado, se realizó una, correlación entre el área de comprensión lectora con hábitos de estudio, resultando correlacionadas la mayoría de las variables que los conforman.

Del eje de aprendizaje se obtuvieron correlaciones de más de 15 variables que lo componen. El análisis muestra que hay correlaciones significativas de tipo positivo a comprender generalmente lo que leen y de tener buenos hábitos de estudio, en otro sentido se encontraron trece relaciones entre comprensión lectora y aprendizaje, de tipo negativo, mientras el estudiante refiere sentir que su estudio mejora con los mapas conceptuales, mencionan no dormir lo suficiente y no tener un lugar fijo para estudiar, lo que les hace no aprobar sus materias, reconocen tener que buen habito de estudio y comprender lo que leen, su aprendizaje es para su vida profesional más significativo y que puedan resolver problemas o situaciones que se le presentaran en su profesión.

Del eje de aprendizaje, en correlación con la comprensión lectora se encontraron cinco variables de tipo positivo. Lo que muestra que en la medida que mejora su aprendizaje es debido a que comprende más lo que lee y fomenta su hábito de estudio repercutiendo en un mejor promedio de carrera, satisfacción personal y de autoestima. 
Respecto al análisis factorial exploratorio en el primer componente se integró con 18 reactivos y se etiquetó con el nombre de confianza en el aprendizaje, este constructo mide la intensidad en que los estudiantes de educación superior consideran el estudio como superación personal. También implica el aprender cosas nuevas como confianza en la capacidad de aprender, así como resultados satisfactorios cuando se ponen a estudiar. Este componente explica el $13 \%$ de la varianza total de la estructura.

El cuarto y último componente de esta estructura, se nombró disciplinas al trabajar, este constructo mide la manera en que el estudiante de educación superior concibe el trabajo en equipo para socializar sus ideas e intercambiar puntos de vista con sus compañeros el nivel de compromiso con sus deberes académicos. Abarca un $6 \%$ de la varianza total explicada y se integró por diez reactivos. Dando respuesta al objetivo tres de investigación que se refiere a desarrollar estrategias de aprendizaje conceptual $\mathrm{y}$ procedimental como herramientas en el proceso educativo. Cuando los estudiantes de educación superior conocen cuál es su estilo de aprendizaje, aplican las técnicas de estudio más convenientes a este estilo y adquieren hábitos de estudio, aprovecharan de manera óptima su aprendizaje y si su maestro conoce dichos estilos, entonces los podrá orientar e inducir a que apliquen esas técnicas y adquieran dichos hábitos.

En relación a la respuesta del objetivo cuatro que se refiere a proponer cambios en la estrategia de aprendizaje que mejoren la comprensión lectora y los hábitos de estudio de estudiantes de educación superior, para obtener mejores resultados académicos.

Cuando el rendimiento académico no es el esperado, es el momento de cambiar de estrategia. Se les dan a conocer las diferentes estrategias y su manera de llevarlas a cabo. Se recomiendan talleres de lectura guiada, mejorar la comprensión lectora, un lugar fijo para estudiar, dormir ocho horas, alimentación adecuada para estar sanos, aplicar técnicas que les motiven y mejorar su autoestima.
La mayoría de las variables de los tres ejes de investigación tienen una correlación positiva, lo que indica que en la medida que los estudiantes de primer semestre de educación superior comprenden lo significativo de sus temas de aprendizaje, sienten que pueden terminar su licenciatura con éxito y aspirar una Maestría, Doctorado y muchos el PostDoctorado. Lo que fomentaría la investigación en las Universidades de Educación Superior.

Comprensión lectora: es la capacidad para entender lo que se lee, tanto en referencia al significado de las palabras que forman un texto, como con respecto a la comprensión global del texto mismo. Aprendizaje: consiste en adquirir conocimientos de cualquier índole que antes no se poseían. Hay ciertas capacidades que son innatas, pero que necesitan desarrollarse, por medio de la adquisición de información, y esta información proviene del aprendizaje.

\section{Referencias}

Acosta, S., y Acosta, R. (2010). Los mapas conceptuales y su efecto en el aprendizaje del conocimiento biológico. Omnia, (16) 2, 209225.

Aguilera, J. R., Castillo, A., y García, J. (2007). Percepción de los roles docente-estudiante: problema que influye en la calidad de la enseñanza. Innovación Educativa, (7) 38, 53-76.

Álvarez, D., Colorado, H., y Ospina, L. (2010). Estrategias docentes para un aprendizaje significativo. In Didáctica de las matemáticas, una experiencia moderna. (p. 18). Colombia: Ediciones Elizcom.

Aparicio, J. A., y Hoyos, O. L. (2008). Enseñanza para el cambio de las representaciones sobre el aprendizaje. Universitas Psychologica, (7) 3, 725-737.

Aranda, J. S. (2009). Mimesis y metamorfosis en el acto de leer. Innovación Educativa, (9) 49, 15 20.

Ardilla, R. (2012). Enfoques conceptuales de la psicología en Colombia. Interamerican Journal of Psychology, (46)1, 79-86.

Arrieta, B., Meza, R., y Batista, J. (2008). Interferencia de los neologismos en la comprensión lectora de textos académicos. Laurus, (14)28, 35-53.

DURÁN-FLORES, Ma. Nelly, FARIAS-BRACAMONTES, Juan Carlos, GARCÍA-GARCÍA, Jesús Alberto y CU-FARFÁN-LÓPEZ, Julio. Comprensión lectora y hábitos de estudio en estudiantes de educación superior, desde el aprendizaje conceptual y procedimental. Revista Teoría Educativa. 2020 
Asensio, M., Carretero, M., y Pozo, J. (1997). La enseñanza de las Ciencias Sociales. Madrid: Visor.

Ausubel, D., Novak, J., y Hanesian, H. (1962). Psicología educativa (2nd ed.). Trillas.

Barboza, Y. (2008). Los materiales instruccionales: ¿factores obstaculizantes de la comprensión de la lectura? Educere, (12) 42, 589-595.

Barroso, C. (2007). Fortalecimiento de competencias para el estudio vía programas académicos en modalidades educativas innovadoras. Innovación Educativa, (7)38, 5-16. Chirinos, N (2012). Estrategias metacognitivas aplicadas en la escritura y comprensión lectora en el desarrollo de los trabajos de grado. Zona Próxima, (1)17, 142-153.

Cornejo, J. N., Roble, M. B., Barrero, C., y Martín, A. M. (2012). Hábitos de lectura en alumnos universitarios de carreras de ciencia y de tecnología. Revista Eureka sobre Enseñanza y Divulgación de las Ciencias, (9)1, 155-163.

Desarrollo de Capacidades. (2006). España: Ministerio de Educación.

Díaz, E., Albarino, G., y Carrascal, N. (2011). Enfoques de aprendizaje y niveles de comprensión: El aprendizaje universitario en ambientes tecnológicos. Fondo Editorial Universidad de Córdoba.

Díaz, F., y Hernández, G. (2011). Estrategias docentes para un aprendizaje significativo. In Una interpretación constructivista. México: McGraw Hill.

Díaz, F., y Hernández, G. (n. d.). Estrategias docentes para un aprendizaje significativo. Una interpretación constructivista. (2nd ed.). McGraw Hill.

Domínguez, D. A., \& Pérez, M. N. (2009). Internet y el hábito de la lectura en los universitarios. Innovación Educativa, (9)49, 1117.

Echeverría, R. (2005). Ontología del lenguaje. Lorn.
Escalante, L. E., Escalante, Y. I., Linzaga, C., y Merlos, M. E. (2008). Comportamiento de los estudiantes en función a sus hábitos de estudio. Revista Electrónica "Actualidades Investigativas en Educación", (8)2, 1-15.

Espinoza, H. (2000). Estrés y comprensión de la lectura: un estudio etnográfico: Universidad de Carabobo.

Fajardo, A., Hernández, J., y González, A... (2012). Acceso léxico y comprensión lectora: un estudio con jóvenes universitarios. REDIE. Revista Electrónica de Investigación Educativa, (14)2, 25-33.

Ferreiro, E., y Gómez, M. (1982). Nuevas perspectivas sobre los procesos de lectura $\mathrm{y}$ escritura. México: Siglo XXI. Forjando una sociedad del conocimiento sustentable. Innovación Educativa, (5)27, 37-43.

Gabinete Psicopedagógico. (2001). Hábitos de Estudio. Universidad de Granada.

Galicia, J. C., y Villuendas, E. R. (2011). Relación entre hábitos lectores, el uso de la biblioteca y el rendimiento académico en una muestra de estudiantes de psicología. Revista de la Educación Superior, (40)127, 55-73.

Gil, J. (2011). Hábitos lectores y competencias básicas en el alumnado de educación secundaria obligatoria. Educación XX1, (14)1, 117-134.

Gómez, H. (1998). La comprensión de la lectura en el $5^{\circ}$ grado. Tesis. México: Universidad Pedagógica Nacional.

González, K. (2008). Propuesta de un programa para mejorar la comprensión de textos en estudiantes universitarios. Revista Electrónica "Actualidades Investigativas en Educación", (8)2, 1-31.

González, Klency. (2009). Diseño de un programa para mejorar la comprensión de textos en estudiantes universitarios. Revista Latinoamericana de Estudios Educativos, (39)1$2,125-151$.

Ferreiro, E., y Gómez, M. (1982). Nuevas perspectivas sobre los procesos de lectura $\mathrm{y}$ escritura. México: Siglo XXI. Forjando una sociedad del conocimiento sustentable. Innovación Educativa, (5)27, 37-43. 
Insausti, M., y Merino, M. (2000). Educación y tecnologías de información y comunicaciones. Una propuesta para el aprendizaje de contenidos procedimentales en el laboratorio de física y química (Vol. V. 5 N.2). Brasil: En Investigaciones en la Enseñanza de Ciencias.

Luque, D., y Rodríguez, G. (2006). Dificultades en el aprendizaje. Unificación de criterios diagnósticos. España: Consejería de Educación.

Manzano, R., y Pickering., D. (2005). Dificultades en el aprendizaje. Dimensiones del aprendizaje ( $2^{\mathrm{a}}$ ed. ITESO ed.). México: Manual para el maestro

Veracruz, México. Revista Electrónica "Actualidades Investigativas en Educación", (11)3, 1-17. 